\title{
La pandemia desde una perspectiva biopolítica
}

Una exploración sobre la vigencia de los análisis foucaulteanos para pensar la crisis sanitaria en tiempos de covid-19

\section{The pandemic from a biopolitical perspective}

An exploration of the validity of Foucauldian analyzes to think about the health crisis in time of covid-19

\author{
Agustina Alejandra Andrada \\ Doctorado (c) Filosofía \\ Universidad Nacional de San Martín, andradaagustina@hotmail.com
}

\begin{abstract}
Resumen
Este artículo se propone analizar la actualidad de los abordajes foucaulteanos sobre el saber médico para explicar los sucesos acontecidos a raíz de la pandemia del covid-19. Para ello nos centraremos en su definición de la medicina como práctica biopolítica, analizando en qué medida esta continúa siendo una técnica política de intervención con efectos de poder sobre la vida y sobre la muerte de la población. Al mismo tiempo, intentaremos pensar el marco de racionalidad política en el que se inscribe la actual crisis sanitaria, esto es: el neoliberalismo y sus consecuentes formas de "hacer vivir y dejar morir".

Palabras clave: biopolítica, Foucault, pandemia, medicina, neoliberalismo.
\end{abstract}

\begin{abstract}
In this paper, We will try to analyze the actuality of Foucauldian approaches to medical knowledge in order to explain the events that occurred as a result of the COVID-19 pandemic. In order to achieve this, We will focus on Michel Foucault's definition of medicine as a biopolitical practice, analyzing to what extent it continues to be a political technique of intervention with effects of power over the life and death of the population. At the same time, We will try to think about the framework of political rationality in which the current health crisis is inscribed, that is: the neoliberalism and its consequent ways of "making live and letting die".

Keywords: Biopolitics, Foucault, Pandemic, Medicine, Neoliberalism.
\end{abstract}




\section{Introducción}

La crisis sanitaria evidenciada por la pandemia del covid-19 ha hecho emerger en el ámbito de la filosofía política contemporánea múltiples debates en torno a la actualidad de la biopolítica para comprender los hechos que ocurren en el presente. Conceptos como estado de excepción, inmunidad, nuda vida, biopoder, que han sido trabajados por filósofos como Agamben, Esposito y Michel Foucault, adquieren hoy una gran relevancia y una nueva significación.

Desde un primer enfoque, se podría pensar que el tratamiento de la pandemia se circunscribe al abordaje de la medicina, la inmunología y la infectología. Sin embargo, la filosofía política aparece para echar luz sobre las implicancias sociales, culturales y políticas de esta crisis, así como también para establecer las condiciones histórico-políticas que le dieron origen.

Por consiguiente, este trabajo parte del supuesto de pensar a la filosofía en un vínculo estrecho con el presente. Concibiéndola como una herramienta para diagnosticar la realidad y postular en función de estos diagnósticos nuevas formas de ser y de habitar la actualidad. Esto significa que tomamos como principio teórico la ontología crítica del presente y de nosotros mismos postulada por Michel Foucault en el ¿Qué es la ilustración? En este texto el autor nos dice: "El discurso [filosófico] tiene que volver a tomar en cuenta su actualidad, por una parte, para recuperar allí su propio lugar, por otra parte para decir su sentido, por último para especificar el modo de acción que es capaz de ejercer en el interior de esa actualidad." (Foucault, 1996, p. 71). De modo que, llevar a cabo esta metodología foucaulteana para interrogar a los acontecimientos que rigen la contemporaneidad implica retomar la pregunta sobre quiénes somos nosotros hoy, qué es lo que le da sentido al presente, a los saberes y las prácticas.

Y en este sentido, consideramos que no es casual que Agamben (2020) reúna todos sus trabajos sobre la pandemia bajo el título ¿En qué punto estamos? Este cuestionamiento evidencia el compromiso de la filosofía política con el presente porque lo lleva a preguntarse por las transformaciones del paradigma político ocurridas en los últimos años y potenciadas por las medidas de excepción a partir de la emergencia sanitaria. Al respecto el filósofo italiano afirma:

“...tendremos que plantearnos seriamente la única pregunta que importa, que no es, como los falsos filósofos han estado repitiendo durante siglos, -de dónde venimos- o -a dónde vamos-, sino simplemente -en qué punto estamos-. Esta es la pregunta que debemos tratar de responder como podamos y dondequiera que estemos, pero en cualquier caso con nuestras vidas y no sólo con las palabras" (Agamben, 2020, pp. 26-27). 
Saber en qué punto estamos implica entonces dar cuenta de las formas que adquieren las prácticas de gobierno en el presente. De modo que este artículo persigue estos objetivos teóricos postulados tanto por Foucault como por Agamben.

Ahora bien, el eje de análisis de este escrito radica en mostrar la vigencia de los abordajes foucaulteanos sobre la medicina como práctica biopolítica. En la última clase de Defender la sociedad, nuestro autor sostiene que: "La medicina va a ser una técnica política de intervención con efectos de poder propios. La medicina es un saber/poder que se aplica, a la vez, sobre el cuerpo y sobre la población" (Foucault, 2014, p. 228). Siguiendo estas afirmaciones, nos preguntamos: ¿Cuál es el estatuto político de la medicina en un presente en estado de pandemia? ¿Qué modalidad adquieren las prácticas de "hacer vivir y dejar morir" en esta crisis sanitaria? Es decir, ¿cuáles son actualmente sus modos de intervención y cuáles sus efectos de poder sobre el cuerpo y sobre la población?

De manera que, a lo largo de este artículo buscaremos responder a estos cuestionamientos tomando como base los conceptos foucaulteanos sobre el saber médico, utilizándolos como herramientas para interpretar la actual situación sanitaria. Nuestra conjetura de base será mostrar que, más allá de las modificaciones dadas en las investigaciones médicas y en los dispositivos de salud, la pandemia nos muestra que la medicina continúa siendo una práctica biopolítica con efectos sobre la vida y sobre la muerte de la población.

Por esto mismo, nuestro primer punto de exploración se centrará en analizar no solamente el estatuto político del saber médico sino además de la noción de pandemia. Mostrando cómo ya en El nacimiento de la clínica Foucault (2008) daba cuenta de las implicancias políticas y de los efectos sobre las conductas de la población dados por el tratamiento y control de las epidemias. Una vez expresadas estas vinculaciones entre los saberes médicos y las prácticas políticas, procederemos a abordar nuestro segundo punto en donde nos abocaremos a investigar el modo en que la gubernamentalidad neoliberal, que prima en el presente, se sirve del saber médico para llevar cabo sus finalidades de gobierno. Este segundo núcleo de análisis parte del hecho de postular al neoliberalismo como el marco de racionalidad política que determina los mecanismos, las finalidades y los efectos del dispositivo biopolítico en nuestra actualidad. 


\section{La medicina y la pandemia como política}

A lo largo de su dossier sobre el coronavirus, Agamben hace hincapié en la connotación política del concepto de pandemia. Al respecto el autor sostiene: "La palabra griega epidemia (de demos, el pueblo como entidad política) tiene un significado político inmediato. Lo más peligroso es confiar a los médicos y a los científicos decisiones que son en una última instancia éticas y políticas" (Agamben, 2020, p. 57) Cuestión que reitera y remarca en la mayor parte de sus reflexiones sobre la emergencia sanitaria postulando que la pandemia es el nuevo terreno de la política en términos mundiales. Para el filósofo italiano estamos vivenciando una nueva forma de guerra civil donde el enemigo se ubica dentro de nosotros mismos bajo la forma de un virus y ello nos obliga a vivir en una especie de terror sanitario donde todo se suspende menos la nuda vida. Desde su perspectiva, este miedo al contagio y esta sensación de crisis perpetua conduce a los hombres a reducir su vida a una condición estrictamente biológica abandonando sus dimensiones políticas, humanas, sociales.

Según estos diagnósticos agambeneanos, la pandemia posee una significación política puesto que instaura determinadas formas de vinculación social que poseen como principio de acción el distanciamiento social, como criterio de veridicción a la medicina y como dispositivo jurídico al estado de excepción. Esta tríada integrada por el distanciamiento social, el estado de excepción y la medicina/ciencia son los elementos que constituyen a la "bioseguridad" como nueva forma de gobierno. Este nuevo paradigma político se ha venido gestando en los últimos años ante la crisis de las democracias burguesas, ante la instauración de la medicina como religión y el avance de la tecnología digital en las formas de comunicación entre los hombres.

Ahora bien, dentro de todos estos ejes de análisis nos interesa detenernos brevemente en la cuestión de la religión de la salud puesto que es allí donde la medicina adquiere, según Agamben, un estatuto político. Desde su visión, en Occidente conviven tres sistemas de creencias: el cristianismo, el capitalismo y la ciencia. Dentro de la historia de la modernidad las relaciones entre estas tres religiones han ido mutando, pero, para el filósofo italiano, en la actualidad la protagonista es la ciencia, tomando como disciplina central a la medicina. El virus y, más específicamente, la enfermedad, son pensados como principios malignos que pueden ser curados mediante la intervención benéfica de la medicina. Desde esta lectura, el saber médico posee un carácter de salvación puesto que es quien preservaría esta vida biológica, esta vida desnuda que se ve amenazada ante el avance de la pandemia. Esto evidencia para Agamben que el objeto de acción de la medicina va más allá de la relación médico- 
paciente al incidir sobre las formas de vida, sobre las conductas de los sujetos en pos de su obligación por preservar su salud. Al respecto el autor argumenta:

Si esta práctica cultual [la práctica médica] era hasta ahora, como toda liturgia, episódica y limitada en el tiempo, el fenómeno inesperado que estamos presenciando es que se ha vuelto permanente y omnipresente. Ya no se trata de tomar medicinas o someterse a una visita medica o a una intervención quirúrgica cuando sea necesario: la vida entera de los seres humanos debe convertirse en el lugar de una celebración cultual ininterrumpida en todo momento (Agamben, 2020, pp. 66-67).

Siguiendo lo dicho en esta cita, parecería que no hay conducta humana que se encuentre por fuera de la intervención médica. De hecho, quienes se opongan a sus principios serán considerados un riesgo para la salud del resto de la población.

Una vez exploradas las hipótesis principales de Agamben para interpretar la situación sanitaria actual consideramos necesario mostrar que el rol político de la medicina, su establecimiento como religión, su incidencia más allá del cuerpo de los enfermos y el manejo político de las epidemias es una cuestión que no se circunscribe a los hechos producidos por el covid-19 sino que se trata de una característica inherente a la medicina moderna. Los análisis sobre el saber médico elaborados por Foucault en El nacimiento de la clínica (2008) y en sus conferencias sobre El nacimiento de la medicina social (1999) dan cuenta de ello. De modo que, haremos una breve mención de sus abordajes al respecto para entender la actualidad de los conceptos allí enunciados.

En primer lugar, consideramos importante destacar que uno de los estudios realizados por Foucault sobre la noción de pandemia fue realizado en un capítulo de El nacimiento de la clínica titulado "Una conciencia política". Ya el nombre del capítulo nos da un indicio sobre la postura foucaulteana respecto a la concepción política de la pandemia y de la medicina. Para el filósofo francés, realizar una indagación arqueológica de la mirada médica implica inexorablemente estudiar el momento en que la salud se vuelve un problema político y, en consecuencia, el médico se transforma en un funcionario estatal. Este momento es ubicado por Foucault hacia fines del siglo XVIII cuando se crea la Real Sociedad de Medicina encargada de estudiar los fenómenos epidémicos. Se trata de una red de conocimiento que además de investigar y describir los movimientos de las epidemias bajo un método complejo de observación, indica de forma prescriptiva y bajo el empleo de una "policía médica" los modos de comportamiento adecuados para obtener el control de las enfermedades. Por consiguiente, para nuestro autor, el surgimiento de la medicina moderna implica: “...la definición de un estatuto 
político de la medicina, y la constitución a escala de un Estado, de una conciencia médica encargada de una tarea constante de información, de control y de sujeción...” (Foucault, 2008, p. 51).

En estas reflexiones se puede observar que la medicina es una práctica que va más allá de su vinculación con una patología concreta, es decir, que su saber no termina en la relación con el enfermo en particular, sino que incide en el fenómeno colectivo de la enfermedad y, por lo tanto, en la población en su conjunto. La profesionalización del médico como funcionario público a partir del siglo XVIII se produce sobre el telón de fondo de una política de la salud. Ahora bien, esta política sanitaria requiere de la intromisión médica en espacios que no son necesariamente terapéuticos: ella debe desprenderse de la función socorrista de las enfermedades para estudiar el campo material complejo que constituye a la vida de la población. Como explica Foucault, se trata de: "El desarrollo de tipos de intervención que no son terapéuticos y ni siquiera médicos en sentido estricto, toda vez que incumben a las condiciones y a los modos de vida, la alimentación, la vivienda, el medio, la manera de criar a los niños, etc.” (Foucault, 2012, p. 213). La práctica médica se vuelve entonces una práctica biopolítica y con ello una gestión política que procura regular la vida de la población.

Siguiendo estos diagnósticos foucaulteanos, y en contraposición a la perspectiva de Agamben, podemos advertir que la epidemia como política y la intromisión de la medicina en la vida entera de los seres humanos no es una novedad inherente a las medidas empleadas para controlar los efectos del covid-19. Esta omnipresencia de la medicina data de aquel momento en el que ella se vuelve una herramienta de ejercicio del poder de la modernidad, capaz de intervenir no sólo sobre los cuerpos sino sobre el medio, sobre las conductas, los deseos con el fin de modificar a la vida de la población para “...distribuir lo viviente en un dominio de valor y de utilidad” (Foucault, 2002, p. 136). Y este señalamiento excede la cuestión exegética puesto que sostenemos que omitir el estatuto biopolítico de la medicina, inserto en ella desde sus orígenes, hace perder de vista que se trata de un saber con efectos de poder que ha ido mutando en función de las necesidades gubernamentales y que ha incidido en las formas de vida y de muerte de la población en su conjunto.

Al mismo tiempo, esta referencia de Agamben a la medicina como religión también fue abordada desde la óptica foucaulteana en El nacimiento de la clínica. Al explicar cómo en la modernidad la profesión médica se nacionaliza y el médico se vuelve un instrumento al servicio del Estado, muestra que éste adquiere el rol que antes cumplían los sacerdotes como salvadores de las almas. La medicina obtiene un carácter de salvación en la medida en que debe aliviar los sufrimientos corpóreos de la 
sociedad y, además, porque es la que establecerá cuáles son las acciones correctas e incorrectas para llevar a cabo en función de la ampliación de la salud.

En torno a esta comparación entre el saber médico y la religión, resulta interesante acudir a los análisis de Jean-Claude Monod, en su libro La police des conduites (2010), donde muestra que esta nueva concepción de la salud como asunto político parte de una mutación ocurrida en el plano de los mecanismos de poder. Tal como explica en su texto:

El fortalecimiento de la autoridad médica se inscribe bien en la transformación de los modos de regulación en las sociedades modernas, que tienden, según Foucault, a funcionar cada vez más según la norma y cada vez menos según la ley. Si la ley refiere en primer lugar a la religión, la norma es más apropiada en las sociedades donde la ciencia se convierte en la fuente de la verdad generalmente reconocida. [...] La pareja religión-derecho, unidas desde la Edad Media a la época clásica a través de formas de enunciación (condenas, reglas) y de poder (tribunales, castigo), son substituidas así por la pareja medicina-norma (Monod, 2010, p. 52).

En consecuencia, la medicina aparece ligada al advenimiento de la norma como herramienta de poder y sustituye a la doctrina religiosa anclada en el derecho. A partir de este momento, el objetivo del poder se halla en la salud de los cuerpos y ya no en la salvación de las almas. La medicina se vuelve la copia secular de la actividad sacerdotal al velar por el bienestar del cuerpo, entendido como cuerpo social. Como aclara Foucault: “...la palabra "cuerpo” no hay que entenderla de manera simplemente metafórica: se trata en efecto de una materialidad compleja y múltiple que comporta, además de los cuerpos de los individuos, el conjunto de los elementos materiales que aseguran su vida, constituyen el marco y el resultado de su actividad y permiten los desplazamientos y los intercambios" (Foucault, 2012, p. 217). De allí que, para alcanzar su meta, el saber médico deberá aplicarse a los múltiples aspectos que constituyen a esta naturaleza compleja del cuerpo social, que no es más que un sinónimo del concepto de población.

De modo que, podemos pensar que la medicina es desde el siglo XVIII un mecanismo de veridicción de la sociedad, entendiendo por veridicción el “...conjunto de reglas que permiten, con respecto a un discurso dado, establecer cuáles son los enunciados que podrán caracterizarse en él como verdaderos o falsos" (Foucault, 2004, p. 53). De allí que, Foucault (2008) sostenga que el médico se ha vuelto un "consejero y un experto" al indicarle a la sociedad los modos de vida correctos para su pleno desarrollo. El saber médico podrá decirnos cuál es el modo indicado de alimentarnos, cuántas horas debemos dormir, cómo debemos criar a los niños en pos de su bienestar futuro, cómo debemos 
relacionarnos con los otros, qué hábitos son los indicados para potenciar nuestra capacidad productiva, etc.

Si entendemos que la medicina es una práctica biopolítica tenemos que afirmar que se trata de un saber que "hace vivir y deja morir". Es decir, que ella le permite al poder apropiarse de la vida y de la muerte en tanto entidades naturales para regularlas en pos de sus intereses. La eficacia de sus prácticas le posibilita al biopoder la creación permanente de nuevas formas de vida y, por consiguiente, de muerte. Los cambios en las tasas de mortalidad y en las expectativas de vida del cuerpo social responden al carácter productivo de este saber-poder que genera continuamente nuevas naturalidades.

Ahora bien, tomando estos análisis como herramientas para pensar el rol de la medicina en nuestro presente y comparándolos con el diagnóstico agambeneano sobre las implicancias políticas de la pandemia podemos afirmar que las mutaciones en la profesionalización médica siguen el modelo de desarrollo impuesto a partir del siglo XVIII. Hoy podemos ver más que nunca que el saber médico continúa siendo una actividad normalizadora que interviene en la sociedad en su totalidad, a punto tal de establecer cómo debemos vincularnos con nuestros afectos, cómo debemos llevar a cabo las reglas de higiene, de qué forma movilizarnos, etc. De esta manera, los cambios ocurridos en sus mecanismos de acción a partir de la pandemia responden a una exacerbación de la racionalización médica expresada en sus inicios.

No obstante, luego de mostrar que actualmente la medicina sigue siendo un saber-poder que interviene sobre la vida y sobre la muerte de la población debemos establecer el marco de racionalidad política en la que ésta se inscribe. Sabemos que los límites y las metas de gobierno están fijados por parámetros de tipo economicistas que distribuyen la vida bajo criterios de valor y de utilidad. Asumiendo que la gubernamentalidad que rige en el presente es el neoliberalismo necesitamos pensar su vinculación con las prácticas médicas. Las formas de proceder de la medicina frente al tratamiento de la pandemia responden a estos intereses de gobierno en donde el criterio de acción se funda en los imperativos de mercado.

\section{El tratamiento de la pandemia bajo las prácticas neoliberales de gobierno}

Continuando con nuestro objetivo de análisis que es pensar la vigencia de los abordajes foucaulteanos sobre la medicina para diagnosticar a este presente atravesado por el coronavirus y asumiendo que nos encontramos ante un saber médico que opera como herramienta de ejercicio del 
biopoder, nos detendremos en este punto a analizar los intereses de gobierno que actualmente subyacen en sus prácticas y cuáles son sus efectos en la vida y en la muerte de la población.

En El nacimiento de la biopolítica Foucault pone de manifiesto que sólo podrá comprenderse lo que es la biopolítica si se estudia en qué consiste el régimen liberal y neoliberal de gobierno. Allí deja en claro que no se trata simplemente de teorías económicas sino de tecnologías especificas para gobernar la sociedad. Estas son las economías generales del poder en las que se inscriben las prácticas biopolíticas donde el mercado se ubica como criterio de verdad y establece los objetivos de maximización de la vida. Nos interesa en este punto retomar la descripción que el autor hace sobre el modo de intervención de la gubernamentalidad neoliberal en la que la sociedad entera adopta la forma empresarial y cada sujeto se convierte en un empresario de sí mismo. El concepto de capital humano muestra, según Foucault, cómo los individuos se conciben a sí mismos bajo la lógica de competitividad del mercado y piensan todas sus acciones mediante criterios economicistas. El resultado de la intervención neoliberal es un sujeto que calcula cada una de sus decisiones en función de mantenerse en situación de mercado (Foucault, 2004).

Frente a esta descripción del neoliberalismo y vinculándola con las prácticas médicas nos preguntamos: ¿cómo la razón neoliberal se sirve actualmente del saber médico para alcanzar sus finalidades de gobierno? ¿Cómo hoy la medicina es atravesada por los criterios de valor y utilidad del mercado? ¿De qué manera influyen los objetivos neoliberales en las formas de tratamiento de la pandemia?

Para responder a estos cuestionamientos, es necesario advertir que una de las consecuencias que Foucault remarca de la intervención de los imperativos de mercado en las prácticas médicas reside en el hecho de convertir a la salud en un objeto de consumo. Desde el siglo XVIII la medicina puede ser concebida como un problema económico en la medida en que produce fuerza de trabajo y garantiza la fuerza laboral mediante la medicalización. Sin embargo, en el presente, bajo las formas economicistas de gobierno, ella no sólo asegura la productividad de los cuerpos, sino que se constituye como fuente de riqueza en sí misma. El saber médico al ampliar su capacidad productiva y mejorar sus técnicas de intervención de la vida se convierte en una actividad ligada al mercado económico. La salud se vuelve un deseo que transforma a los pacientes en consumidores que eligen y usan activamente la medicina para maximizar sus vidas. Como resume Foucault: "La salud en cuanto se convirtió en objeto de consumo, que puede ser producido por laboratorios farmacéuticos, médicos, etc., y 
consumidos por otro -los enfermos posibles y reales-adquirió importancia económica y se introdujo en el mercado" (Foucault, 1976, p. 165).

La medicina en las formas neoliberales de gobierno se vuelve una práctica de poder capaz de introducir al cuerpo humano en el mercado bajo la premisa del consumo de la salud. Los pacientes comienzan a concebirse a sí mismos como enfermos potenciales, como cuerpos imperfectos capaces de ser mejorados mediante la implementación de estas nuevas técnicas terapéuticas.

En relación a esta problemática de la inserción de lo corpóreo en el ámbito del mercado, el establecimiento de la noción de información genética, los avances en la inmunología y las formas de intervención médica como la estética o la ortopedia cambian la mirada del enfermo sobre su propio cuerpo. Todos los seres humanos seríamos portadores asintomáticos de una enfermedad interna posible de ser curada. Tal como sostiene Flavia Costa (2010): “...todos los cuerpos, incluso los más bellos, armónicos y deseables pueden ser corregidos porque no hay un modelo humano a alcanzar: todos son imperfectos. [...] el cuerpo debe protegerse de sí mismo, de su corrupción natural, saliendo de sí, duplicándose con el propósito de durar" (pp. 161-162). Por consiguiente, se debe procurar de forma constante el bienestar y el mejoramiento del propio cuerpo. La enfermedad es un derivado de la desatención y del poco cuidado del individuo sobre sí. Un sujeto será saludable en la medida en que haya invertido lo suficiente en el mercado de la salud. Las tecnologías médicas son utilizadas por la población como inversiones que le permitirán modificar a futuro su estado de salud.

En efecto, se comienza a juzgar negativamente a aquellos que no han invertido en la salud, que no han adoptado una posición activa respecto a la mejora de sus condiciones de vida. Es decir: "La persona se enferma porque no ha invertido suficientemente en sí mismo.” (Costa, 2010, p. 162) Si la salud es un objeto de consumo, negarse a su consumición es vedarse a sí mismo la posibilidad de un futuro mejor. Pero al mismo tiempo, esta no inversión en el mercado de la salud representa una amenaza para el cuerpo social en general. Así, aquellos que no ingresen en este juego económico de la salud serán vistos como egoístas sociales porque su desatención puede traer consecuencias sanitarias a toda la atmosfera social. Con todo, vemos que las actividades concernientes a la salud de los individuos aparecen como una forma de ampliar, conservar y utilizar el capital humano. Tal como afirma Foucault: "Es preciso por lo tanto repensar todos los problemas de protección de la salud, de la higiene pública como elementos capaces de mejorar o no el capital humano" (Foucault, 2004, p. 53). 
Todos estos análisis que ligan la racionalidad neoliberal con las prácticas médicas pueden verse evidenciados en muchas situaciones ocurridas por la crisis sanitaria actual. En primer lugar, respecto a cómo la medicina se ha vuelto un objeto de consumo postulamos como ejemplo la problemática ocurrida en la Argentina en la relación entre la medicina pública y la medicina privada al inicio de la pandemia. $\mathrm{Al}$ aparecer los primeros casos de coronavirus en diferentes provincias del país el ministro de salud de la nación, Ginés González García, propuso que el Estado tomara a su cargo el manejo de las clínicas privadas para poder hacer uso de las salas de internación y derivar a los infectados independientemente de su cobertura médica. Inmediatamente anunciada la idea y tras una reunión con las autoridades representantes del sector privado de la medicina dicha propuesta fue descartada. El rechazo de un decreto como este se dio por la amenaza de los principales empresarios de la salud a emitir recursos de amparo para frenar la medida y cuidar los intereses de sus consumidores. En este ejemplo se puede ver cómo el consumo de la salud y los criterios economicistas se mantienen aún en una situación de emergencia como la vivida en nuestros tiempos. Desde la visión privada de la medicina, sólo podrá ser atendido aquel que haya invertido en su salud, aquel que haya abonado por su internación, por su cuidado. Aquí el "dejar morir" aparece bajo el modo de la desatención y el abandono sobre aquellos que no puedan tomar a la salud como un elemento de ampliación de su capital humano.

A su vez, estas reflexiones se conectan con lo que Agamben (2020) llama "obligación de la salud”. Según el filósofo italiano, los discursos de los diferentes Estados en torno a las formas de cuidado, confinamiento y distanciamiento social traen consigo una obligación jurídica de la salud que debe ser cumplida sin importar cuál sea su costo. Frente a estos análisis, consideramos que el imperativo de la salud no es una novedad inherente a las medidas tomadas por el tratamiento de la pandemia del covid-19. Prueba de ello son los señalamientos foucaulteanos que aseguran que ya "En el siglo XIX aparece en todos los países del mundo una copiosa literatura sobre la salud, sobre la obligación de los individuos de garantizar su salud, la de su familia, etc." (Foucault, 1976, p. 2). De modo que, nuevamente vemos en las situaciones sanitarias producidas por la pandemia una exacerbación de las formas de intervención médica dadas en la modernidad. Como veíamos al mencionar los análisis de Flavia Costa (2010), los avances en las investigaciones médicas muestran que los sujetos se conciben a sí mismos como imperfectos y todos aquellos que no hayan potenciado sus condiciones de vida no sólo serán pensados como responsables de sus propias enfermedades sino, además, como un peligro para la salud de la población. Esto evidencia que el consumo de la salud no es una opción de los sujetos sino una obligación (o auto-obligación) en la medida en que su cuidado 
produce efectos en el cuerpo social. En este sentido, la obligatoriedad de la salud se ha venido gestado desde el inicio de la medicina moderna, pero se ha potenciado por el afianzamiento de las políticas neoliberales como única forma posible de gobierno.

No obstante, coincidimos con Agamben en que este deber de la salud expresado en las medidas de distanciamiento social, aislamiento obligatorio y reglas de higiene implica “...descargar sobre los ciudadanos la gravísima responsabilidad en que los gobiernos han incurrido al desmantelar el sistema sanitario" (Agamben, 2020, p. 24). Este tipo de medidas busca frenar la cantidad de contagios y evitar el colapso de los hospitales de las diferentes regiones de los países afectados. Esto se da sobre el trasfondo de una desinversión de las políticas públicas que ha imposibilitado que el sistema de salud pueda hacer frente a una emergencia como lo que estamos vivenciando. Por ejemplo, en Argentina en el 2018 el ministerio de salud fue disminuido a una secretaría a cargo del departamento de desarrollo quitándole no solamente autoridad en sus decisiones sino reduciendo los recursos económicos destinados a ampliar el material sanitario. De todas maneras, este desmantelamiento del sistema sanitario no se circunscribe a las políticas neoliberales de América Latina. Tal como expone Renaud Lambert y Pierre Rimbert en la revista Le Monde Diplomatique:

En 1980 Francia disponía de once camas de hospital por cada mil habitantes. Hoy no hay más de seis. En Estados Unidos, las 7,9 camas por mil habitantes inventariadas en 1970 se reducen a 2,8 en 2016. Según la Organización Mundial de la Salud, Italia contaba con 922 camas dedicadas a los "casos serios" por 100.000 habitantes en 1980, contra 275 treinta años más tarde (Lambert y Rimbert, 2020).

De modo que, los efectos mortíferos producidos por el coronavirus son potenciados por un sistema de salud empobrecido por las medidas neoliberales de reducción de presupuesto acompañadas de un discurso que coloca en los individuos la responsabilidad de su salud. Como explica Flavia Costa, con la gubernamentalidad neoliberal aparece una "biopolítica informacional" que es una forma de relación entre el gobierno y los ciudadanos “...en la que se promueve que las personas estén informadas acerca de que deben cuidarse, y acaso cómo, mientras se desatienden y desfinancian las infraestructuras materiales, los equipamientos, la investigación científica y la formación de los trabajadores y profesionales de la salud para tratar con nuevas, y no tan nuevas, enfermedades" (Costa, 2020, sec. 2). En este sentido, la regulación de la pandemia por parte del neoliberalismo se basa en estos mensajes informacionales que proponen apelar al cuidado individual para no interrumpir el juego económico del mercado. Las estadísticas aparecen en este punto como una forma de justificación que explica que el porcentaje de mortalidad producido por el covid-19 no amerita la suspensión de la lógica 
de competitividad de mercado. Los sujetos, pensados como unidades-empresas, deberán velar por su propia salud, deberán preservar su capital humano y el Estado sólo intervendrá en los momentos en que sus finalidades economicistas se vean amenazadas. Es decir, cuando los índices de mortalidad comiencen a afectar los índices económicos.

En estos análisis puede verse que estamos ante una nueva modalidad del "dejar morir" biopolítico. En Defender la sociedad Foucault sostenía que esta fórmula que explica la relación entre el poder y la muerte no significa necesariamente un “....asesinato directo, sino también todo lo que puede ser asesinato indirecto: el hecho de exponer a la muerte, multiplicar el riesgo de muerte de algunos o sencillamente, la muerte política, la expulsión, el rechazo, etcétera” (Foucault, 2014, p. 231). Desde nuestra perspectiva de análisis, el juego social guiado por los criterios de mercado conduce a una parte de la sociedad a su expulsión y, por lo tanto, a un asesinato indirecto. Aquellos que no puedan invertir en su salud quedándose en sus casas, estableciendo los cuidados de higiene, manteniendo el distanciamiento social estarán expuestos al contagio y así se multiplicará el riesgo de muerte de una parte de la población. El "dejar morir" es un resultado del renunciamiento del gobierno a las políticas de bienestar e inserción social. Como resume Foucault: "Nuestros sistemas de cobertura social imponen un modo de vida determinado al que los individuos deben someterse y toda persona que, por una razón u otra, no puede acceder a ese modo de vida se encuentra marginado por el juego mismo de las instituciones" (Foucault, 1991, p. 220).

\section{Conclusión}

A lo largo de este artículo hemos abordado las diferentes nociones empleadas por Foucault en sus análisis sobre el rol biopolítico de la medicina y hemos pensado en qué medida continúan vigentes en la actual situación sanitaria. En este sentido, hemos advertido que el saber médico continúa siendo una estrategia al servicio del poder que adecúa sus prácticas en función de los intereses de gobierno. La medicina es un saber-poder que hace vivir y deja morir, que modifica a la vida y a la muerte en términos poblacionales para distribuir lo viviente en un dominio de valor y de utilidad. La pandemia muestra que la incidencia de las prácticas médicas supera la relación médico-paciente convirtiéndose en un mecanismo de veridicción social.

En línea con esto, hemos mostrado las implicancias políticas de la medicina vinculándola con la gubernamentalidad neoliberal que rige en nuestro presente. En estos análisis vimos cómo hoy los intereses de gobierno se fundan en los criterios de mercado y transforman a la salud en un objeto de 
consumo. Bajo este marco de racionalidad política que legitima el accionar médico observamos un proceso de vaciamiento del sistema sanitario que es acompañado de un discurso informacional de cuidado en donde cada sujeto es responsable de potenciar y preservar su salud. Así, el "dejar morir" aparece ante una forma de gobierno en donde la muerte es pensada mediante índices de mortalidad y sus valores sólo adquieren relevancia en la medida en que éstos afecten sus intereses economicistas. Esta crisis sanitaria ha puesto en evidencia este "asesinato indirecto" producido por la no intervención del Estado en materia de salud, ha mostrado las desigualdades en el acceso al consumo de la medicina, ha dado cuenta que la biopolítica como dispositivo de poder continúa vigente potenciando determinadas formas de vida y abandonando hacia la muerte a aquellas subjetividades que no puedan ampliar por sí mismas su capital humano. 


\section{REFERENCIAS}

Agamben, G. (2020) ¿En qué punto estamos? La pandemia como política. Adriana Hidalgo editora.

Costa, F. (2010). "La vida como información, el cuerpo como señal de ajuste: los deslizamientos del biopoder en el marco de la gubernamentalidad liberal". En Lemm, V. Neoliberalismo y biopolitica, (pp. 161-170). Universidad Diego Portales.

Costa, F. (2020) "La pandemia como accidente normal". Revista Anfibia. http://revistaanfibia.com/ensayo/la-pandemia-accidente-normal/

Foucault, M. (2008) El nacimiento de la clínica. Una arqueología de la mirada médica. Siglo XXI Editores.

Foucault, M. (2012) Elpoder, una bestia magnifica. Siglo XXI Editores.

Foucault, M. (1999) Estrategias de poder. Obras esenciales. Volumen III. Paidos.

Foucault, M. (2008) Historia de la sexualidad 1. La voluntad de saber. Siglo XXI Editores.

Foucault, M. (1976). "La crisis de la medicina o la crisis de la antimedicina". Educación Médica y Salud. 10. $152-170$.

Foucault, M. (2004). Nacimiento de la biopolítica. Siglo XXI Editores.

Foucault, M. (1996). ¿Qué es la ilustración? Ediciones La piqueta.

Foucault, M. (1991). Sabery Verdad. Ediciones La piqueta.

Lambert, R. y Rimbert, P. (2020) "El capitalismo del desastre". Le monde diplomatique. 250. https:/ / www.eldiplo.org/250-ya-nada-sera-como-antes/el-capitalismo-del-desastre/

Monod, J. (2010). La police des conduites. Editions Michalon. 